\title{
A Rare Presentation of Mitral Stenosis
}

\author{
MANDAL SAROJ, ${ }^{1}$ MANJUNATH ${ }^{2}$ DAS SHYMALENDU, ${ }^{3}$ MANDAL DEBASRITA, ${ }^{4}$ KANJILAL SOUVIK, ${ }^{5}$ \\ HAZRA PRAKASH, ${ }^{6}$ PANJA MANOTASH${ }^{7}$
}

\section{Introduction:}

Mitral stenosis of rheumatic origin still looms large in developing countries like ours. Symptoms of such conditions occur when the severity starts increasing or atrial fibrillation develops. They are mainly because of pulmonary venous and arterial hypertension, low cardiac output, as well as embolic phenomena. Our case deals with a rare complication of this common disease.

\section{Case study:}

56 yrs old male of known rheumatic heart disease for many years with moderate symptoms was admitted with complain of respiratory distress of acute onset. He was on regular penicillin prophylaxis, diuretic and rate controlling medicines and had been doing well with those. However few days before admission he had sudden shortness of breath which did not respond as well as it had used to be earlier.

On admission, he was orthopnoeic, having tachycardia with irregular pulse and normal blood pressure. ECG showed atrial fibrillation with $\mathrm{T}$ wave inversion in $\mathrm{V}_{1-3}$ and inferior leads. TTEcho revealed dilated LA, mildly enlarged RV with evidence of rheumatic involvement of mitral valve whose area by planimetry was $1.36 \mathrm{~cm}^{2}$. Transmitral pressure gradient was about $12 \mathrm{~mm} \mathrm{Hg}$ (mean). There was no clot in LAA. LV function appeared depressed with hypokinesia noted in infero-posterior area. Considering the age and the presentation of the patient, $\mathrm{CAG}$ was performed. It revealed $50-60 \%$ stenosis with thrombus in mid RCA with distal cut off and minor plaque in proximal LAD (fig-1).

1. Asst. Professor, IPGMER and SSKM Hospital, Kolkata

2. Md (DM Cardiology 2nd Year), IPGMER and SSKM Hospital, Kolkata

3. MD (Medicine),ESI Hospital, Kolkata

4. MD (Obstetrics and Gynaecology),IPGMER and SSKM Hospital, Kolkata

5. MD (DM Cardiology), IPGMER and SSKM Hospital, Kolkata

6. MD (DMCardiology), Interventional cardiology AMRI hospital, Kolkata

7. Ex Director and Head of Dept. of Cardiology, IPGMER, Kolkata

Correspondence: Dr. Manjunath. MD (DM Cardiology 2nd Year), IPGMER and SSKM Hospital, Kolkata, India. E-mail: gmcmanju@gmail.com.

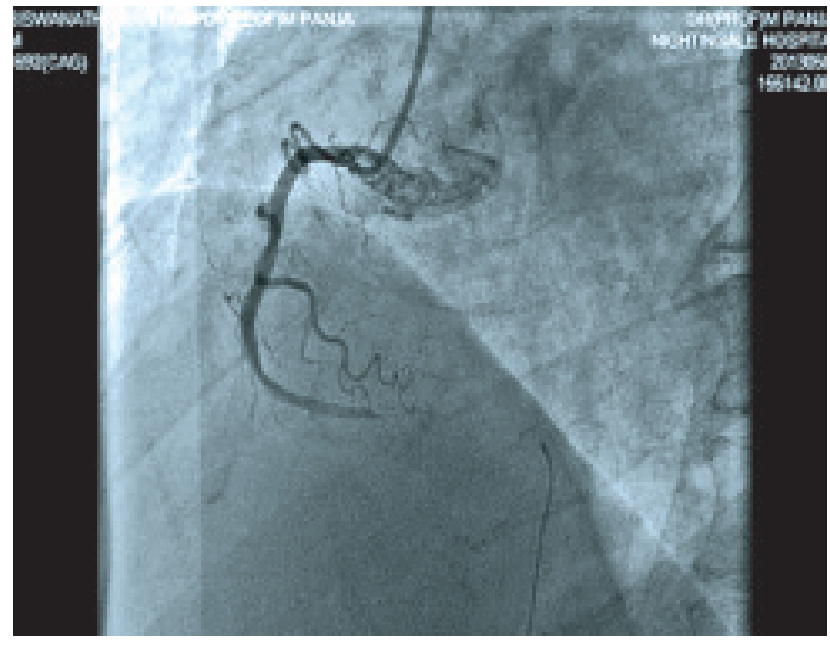

Fig.-1: CAG of RCA showing distal cut off due to thrombus.

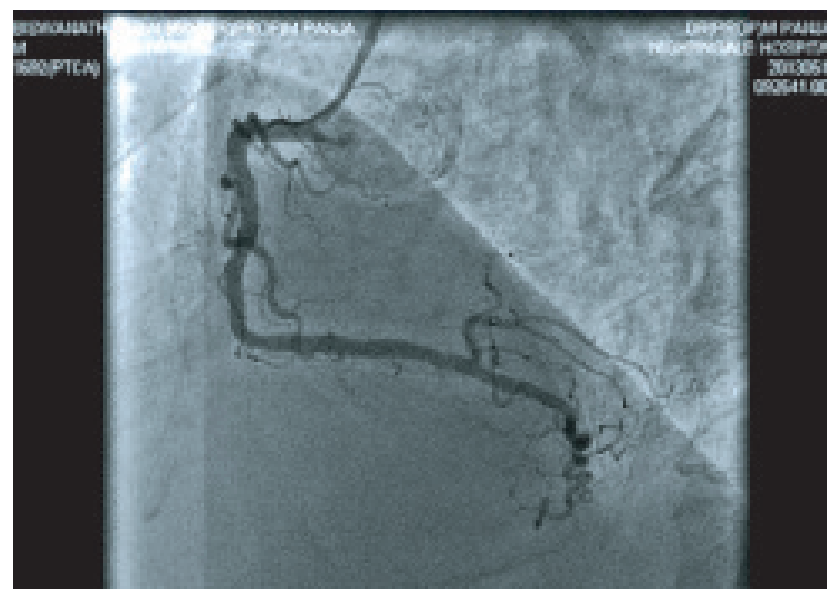

Fig.-2 : final result after dislodgement of thrombus

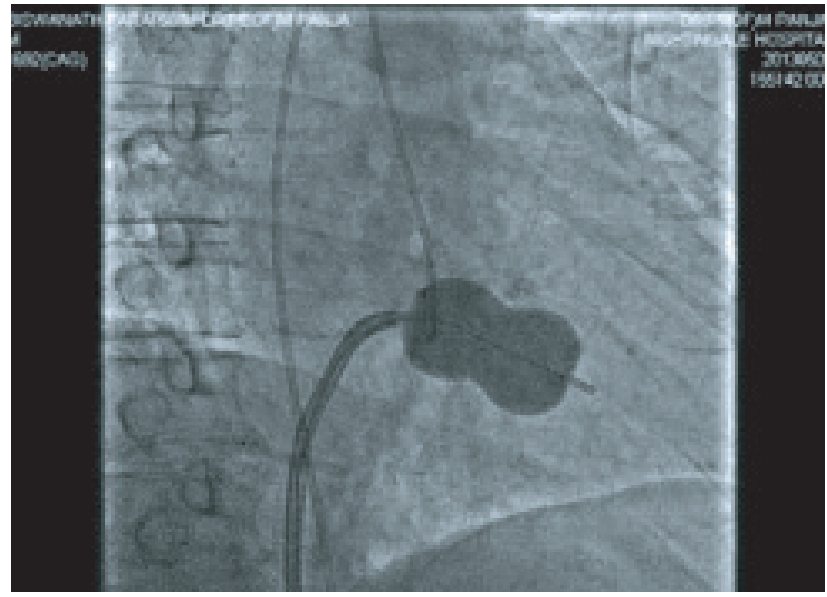

Fig.-3: $B M V$ performed thereafter as usual 
Due to the thrombus in the distal RCA, percutaneous therapy was planned. After an attempt to pass the occlusion with a rather floppy wire (BMW) the occlusion moved slowly a few centimetres more distally; a new stiffer wire (PT2 light support, Medtronic) could not successfully pass the thrombus but pushed it gradually more distally, revealing several small side branches. Because of the very distal location it was not possible to try to remove the thrombus with a suction device. At the location of occlusion and further on in the course ofthe recanalised part of the vessel no coronary abnormalities were seen (figure 2). At this point the clinical suspicion of coronary artery embolism as the cause of the STEMI was high. This angiographic appearance together with the atrial fibrillation was highly suggestive. No further intervention was or could be performed because of the distal location of the occlusion with a relatively small area of myocardium at jeopardy. PTMC was carried out in usual manner thereafter and the patient's condition improved remarkably.A few days later she was dischargedin good clinical condition.

\section{Discussion:}

Acute coronary syndrome is usually brought about by in situ thrombosis of the coronary arteries unlike intracerebral arteries where embolisms make the majority of cases. However, embolism in coronary arteries leading to ACS is possible. Coronary artery embolism is a rare cause of acute myocardial infarction (AMI) and the precise diagnosis remains challenging for the (interventional) cardiologist. The prevalence of this nonatherosclerotic entity remains unknown because of its difficult diagnosis in the acute setting. In general, 4 to $7 \%$ of all patients diagnosed with an AMI do not have atherosclerotic coronary disease at autopsy or coronary angiography. ${ }^{1}$ Furthermore, in another autopsy study 55 out of 419 patients (13\%) showed coronary artery embolic infarcts. ${ }^{2}$ During primary PCI, signs of distal embolisation can be seen by coronary angiography as small peripheral stops and are a marker for a worseprognosis. ${ }^{3}$ There are several case reports of AMI secondary to thromboembolism. In our case, absence of plaque underlying the thrombus and easy dislodgement made us to believe that an embolus, possibly from LA was the culprit.

Coronary embolism is believed to occur due to embolism of LA clot or vegetation of the valves, though it is a rare complication of mitral stenosis.Systemic embolism in patients with MS is caused by left atrial thrombus formation. Although systemic embolization most often occurs in patients with AF, $20 \%$ of patients with MS and a systemic embolic event are in sinus rhythm. ${ }^{4}$
When embolization occurs in patients in sinus rhythm, the possibility of transient AF or underlying infective endocarditis should be considered. However, up to $45 \%$ of patients with MS who are in normal sinus rhythm have prominent spontaneous left atrial contrast (a marker of embolic risk) seen by TEE.

The risk of embolism correlates directly with patient age and left atrial size and inversely with the cardiac output. The consequences of coronary embolism depend on two major factors: the size of the embolus and the size of the lumen of the artery in which it becomes impacted.The smaller the embolus, the greater the chance that it will travel distally to a smaller coronary arterial segmentand less is the likelihood of a myocardial infarction or fatal arrhythmia. ${ }^{5}$

Before the advent of surgical treatment, this serious complication of MS developed in at least $20 \%$ of patients at some time during the course of their disease. Before the era of anticoagulant therapy and surgical treatment, approximately $25 \%$ of all fatalities in patients with mitral valve disease were secondary to systemic embolism. Approximately half of all clinically apparent emboli are found in the cerebral vessels. Coronary embolism may lead to myocardial infarction and/or angina pectoris.

Nowadays, it is obvious that when confronted with a patient with ST-segment elevation myocardial infarction, no time should be wasted and urgent transport for intended primary PCI is crucial.However, when coronary embolism is suspected, interventional techniques using thrombus aspiration devices and intravascular ultrasound become more important. ${ }^{6}$ In the literature, intracoronary thrombolysis and stent implantation are also described as reperfusion strategies, but there is no consensus about the optimal management. Furthermore stent implantation may not be theoptimal recanalisation technique when the underlying pathophysiological mechanism of the myocardial infarction is thrombo embolism.

We report a case of valvular AF with sudden LV dysfunction which we believe due coronary thromboembolism. No direct transoesophageal echocardiography (TEE) was performed which might be seen as a shortcoming, but even absence of thrombus in the left atrial appendage does not preclude a thromboembolism. Furthermore direct TEE does not change the treatment of this patient which consists of aggressive anticoagulation and rate control. We believe that the specific angiographic images and the response of the thrombus to the wire manipulations, together with the clinical presentation are highly specific for coronary embolism.

\section{Conflict of Interest : None}




\section{References:}

1. Waller BF. Atherosclerotic and nonatherosclerotic coronary artery factors in acute myocardial infarction. Philadelphia: Dabis; 1989:29-104.

2. Prizel KR, Hutchins GM, BulkleyBH. Coronary artery embolism and myocardial infarction.Ann Intern Med 1978;88:155-61.

3. Henriques JP, Zijlstra F, Ottervanger JP, de Boer MJ, van 't Hof AW, Hoorntje JC, et al. Incidence and clinical significance of distal embolization during primary angioplasty for acute myocardial infarction. Eur Heart J 2002;23:1112-7.
4. GargRK, Jolly N. Acute myocardial infarction secondary to thromboembolism in a patient with atrial fibrillation. Int $\mathrm{J}$ Cardiol2007;123:e18-20.

5. Roberts WC. Coronary embolism.A review of causes, consequences and diagnostic considerations. Cardiovascular Medicine 1978;3:699-709.

6. Van de Walle S, Dujardin K. A case of coronary embolism in a patient with paroxysmal atrial fibrillation receiving tamoxifen.Int J Cardiol2007;123:66-8. 